\title{
Factores Relevantes en la Elección de los Alimentos de Adultos Mayores en la Provincia del Guayas
}

\author{
Relevant Factors in Food Choice of Senior Citizens in \\ Guayas Province
}

\author{
Miguel Bustamante-Ubilla; ${ }^{1}$ María Lapo-Maza;² María Núñez-Lapo; ${ }^{2}$ Laura Zambrano-Chumo² \\ 1 Facultad de Economía y Negocios, Universidad de Talca \\ 2 Facultad de Especialidades Empresariales, Universidad Católica de Santiago de Guayaquil
}

\section{Resumen}

El presente trabajo determina los factores que inciden en la decisión de los adultos mayores, AM, en la elección de los alimentos. Se valora, en lo esencial, que la alimentación es un aspecto fundamental que incide en la calidad de vida subjetiva del ser humano, en consecuencia, identificar dichos factores resulta un hecho clave. Se aplicó el método de encuesta mediante un cuestionario que recogió datos demográficos y, las percepciones de los AM, respecto de un conjunto de reactivos agrupados en dimensiones que inciden en la decisión de elegir o no los alimentos. Se determinaron, mediante análisis factorial, los factores más relevantes que inciden en la decisión de elección de los alimentos, destacando entre estos, las características del producto, los relacionados con la salud y la economía, la apariencia externa y marca de los productos, la procedencia y finalmente los componentes relacionados con la inocuidad de los productos y su calidad.

Palabras clave: calidad de vida; adultos mayores; alimentación; decisión de compra.

\section{Abstract}

This study determines the factors that influence the decision of older adults, AM, in the choice of food. Essentially, it is valued that food is a fundamental aspect that affects the subjective quality of life of the human being, therefore, identifying these factors is a key fact. The survey method was applied using a questionnaire that collected demographic data and, the perceptions of the MA, regarding a set of items grouped into dimensions that influence the decision to choose or not to choose foods. The most relevant factors that influence the food choice decision were determined through factor analysis, highlighting among these, the characteristics of the product, those related to health and the economy, the external appearance and brand of the products, the origin and finally the components related to the safety of the products and their quality

Keywords: quality of life; elders; food; purchase decision

e-mail: mabu@utalca.c 


\section{INTRODUCCIÓN}

La alimentación en el ser humano es un factor clave para que las personas puedan realizar sus actividades diarias (García, 2002) con la suficiente capacidad de respuesta ante las situaciones que se le presenten (Ortiz y Castro, 2009); sin embargo, para las personas, independientemente de su grupo etario, existen múltiples factores que influyen al momento de realizar la elección de los alimentos para el consumo (Álvarez y Álvarez, 2009; Grunert, et al., 2007). Un caso particular presenta entonces la situación del envejecimiento de la población mundial que avanza en una proporción de $2 \%$ anual, incrementando el número de adultos mayores de 60 años. Se calcula que en el planeta viven 600 millones de personas mayores de 60 años y se proyecta que en el año 2025 se duplicará y en el 2050 ascenderá a 2000 millones de personas (Shamah-Levy, 2008).

Dado que el envejecimiento es un proceso complejo que abarca cambios moleculares, celulares, fisiológicos y psicológicos (Forttes, Massad y Cols, 2009), cobra especial relevancia el hecho de conocer cuáles son los factores que influyen en la elección del tipo de alimentación que cada persona decida para sí durante su vida (Diener et al., 1999); puesto que, la nutrición tiene un rol fundamental (Netuveli, Blane, 2008) mediante la modulación de las funciones de diferentes órganos y tejidos (Forttes, Massad y Cols, 2009), especialmente importante para el grupo etario mayor de 60 años.

En ocasiones, los factores relevantes a la hora de decidir qué alimentos adquirir son los problemas económicos, pero también pueden ser funcionales y psicosociales, los cuales influyen negativamente en la oportunidad de acceso y, posteriormente, en el estado nutricional de los adultos mayores, aún en países con mayor crecimiento (Urteaga, 2012).

Por otro lado, una de las ramas de las ciencias que permite comprender al consumidor, es el Marketing, disciplina que se encarga de observar las necesidades del individuo a través de la investigación de mercado (Aaker y Day, 1996), rama derivada del mercadeo donde se obtiene información a partir de la cual se crea o innova ciertos productos con el fin de mantener la participación de mercado (Kinnear y Taylor, 1993).

En las últimas décadas, el comportamiento del consumidor evidencia que el mismo toma una postura más consciente con respecto a los productos que compra y por ende que consume (Aaker y Day, 1996). De la misma manera, se puede resaltar que hay mucha discusión respecto del rol que ejerce la publicidad y, en general, el marketing en estas decisiones (Laudon y Albert, 1991). Este efecto se produce dado que los alimentos ejercen un impacto en la salud de los consumidores (Leite Nacif \& Ishimoto , 2001). En el caso de los adultos mayores, su alimentación se encuentra restringida por múltiples condiciones (Forttes, Massad y Cols, 2009) que los convierte en un público selectivo al momento de desarrollar productos dentro de la plaza donde se encuentren habitando (Stanton, 1995).

Los adultos mayores deben considerar muchos aspectos antes de poder consumir sus alimentos que van desde: salud, niveles de colesterol, azúcar, grasas que cada uno de los alimentos lleguen a contener (Mendoza, 2013); e inclusive enfermedades como la diabetes e hipertensión, por lo que la alimentación se torna en un aspecto relevante al momento de garantizar la buena salud de los adultos mayores (Forttes, Massad y Cols, 2009).

En países como Chile, existe un bajo porcentaje de éxito - inferior a 30\% - en obtener cifras dentro de los límites actuales de normalidad de la presión arterial en pacientes hipertensos, por lo que se puede inferir la magnitud del problema, cuando se sabe que las complicaciones aumentan con la edad y el envejecimiento; lo cual puede ser regulado mediante factores protectores (Jenkins, 2005). Adicionalmente, es sabido que los adultos mayores toman en consideración aspectos claves para su propio bienestar (Osorio, et al, 2011) como textura, sabor, problemas de masticación, salivación y deglución, discapacidad, minusvalía, deterioro sensorial e hipofunción digestiva (Restrepo, 2006). 
A consecuencia de lo señalado, uno de los factores importantes dentro de la alimentación que las personas decidan para sí se ubica en la promoción comercial de alimentos (Olivares, Lera M., \& Mardones, 2011), situación que resulta en un desafío para los responsables de la elaboración de las estrategias comerciales (Stanton, 1995), como es el hecho de comprender cuáles son los factores que motivan a los individuos a comprar o elegir ciertos alimentos, siendo el caso de los adultos mayores uno de los temas de mayor relevancia, considerando las tendencias poblacionales de éstos a futuro (Shamah-Levy, 2008), por una parte, porque existen variables relacionadas con salud, disponibilidad, capacidad de pago, enfermedades, entre otros factores, que vuelve a este grupo etario más vulnerable en torno a la elección de los alimentos que consume (Jenkins, 2005).

Una de las principales preocupaciones de las compañías que desarrollan actividades de comercialización de productos de consumo es la de ser aceptados y respetados por la sociedad en la que desarrollan su actividad (Laudon y Albert, 1991). La confianza de los consumidores en dichas compañías es fundamental para la actividad de comercialización de productos y servicios, en un mercado que cada día resulta más competitivo (Gonzalez, 1995).

Sobre la base de lo señalado en los párrafos anteriores, esta investigación determina los factores que resultan importantes para los adultos mayores del Guayas en el momento de tomar la decisión de acceso o compra de los productos que consumen.

Finalmente, los resultados esperados, se estima contribuirán a orientar las decisiones de los agentes relacionados con la producción, comercialización y elaboración de las estrategias comerciales y poder detectar oportunidades dirigidas a atender las necesidades de este grupo objetivo de adultos mayores que, en algunos casos, no ha sido considerado un segmento de clientes interés para las empresas que ofertan bienes de consumo.

\section{MÉTODO}

La investigación realizada es de orden cuantitativo, de tipo exploratoria (Hernández-Sampieri y Mendoza, 2018). Los participantes de la investigación fueron un total de 817 adultos mayores, donde se seleccionó a una muestra representativa (Eyssautier, 2006) de la provincia del Guayas del Ecuador, al tomar aleatoriamente adultos mayores de distintos cantones de la provincia, como: Guayaquil, Durán, Naranjal, entre otras.

Se aplicó un cuestionario para recolectar información demográfica y las percepciones que determinan las decisiones de compra por parte de los AM, totalizando 40 reactivos, tomando en cuenta además algunas dimensiones relacionadas con la alimentación, enfermedades, estado de salud, conducta, etc. (Johnson y Wichern, 2007).

Para el análisis de datos se recurrió al uso de herramientas tecnológicas (Walpole et al., 2007) además de programas de análisis tales como IBM SPSS Statistics Versión 22.0, la cual permitió realizar el análisis factorial y análisis de conglomerados (Eyssautier, 2006), conjuntamente con el uso la herramienta de Microsoft Excel 2013 para una mejor interpretación de datos.

\section{Procedimiento}

Los adultos mayores fueron entrevistados en sus lugares de residencia (Hernández-Sampieri y Mendoza, 2018) y los datos fueron organizados en una base de datos con respuestas recogidas en escala de Likert (Likert, 1932), considerando categorías tales como las siguientes: Indique el grado de importancia de los siguientes aspectos de un alimento al momento de comprarlo, sobre la base de 21 reactivos que inciden en la decisión de compra de alimentos por parte de los AM (Walpole et al., 2007).

Se aplicó el método de análisis factorial, considerando el test de esfericidad de Bartlett cuyo propósito fue probar la hipótesis nula de que las variables se encuentran interrelacionadas, situación que se evidencia con un nivel de significancia aceptable si esta resulta $\leq 5 \%$ (Freiberg, et al., 2013). 
Se aplicó el método de extracción de componentes principales, con rotación Varimax, normalización Kaiser y previendo convergencia, la cual fue lograda en la $9^{\circ}$ iteración, finalmente determinando la solución factorial mediante la matriz de componentes rotados (Johnson y Wichern, 2007). Se determinaron las cargas factoriales para cada uno de los factores resultantes y que soportan las etiquetas colocadas a cada una de dichas dimensiones de análisis.

Para la aplicación de la técnica de la técnica de análisis factorial, se requirió el uso del programa estadístico informático SPSS versión 22.0, analizando los 21 ítems propuestos. Posteriormente, los resultados se exportaron a Microsoft Excel 2013, recopilando la información en factores que caracterizan las decisiones de los adultos mayores.

\section{RESULTADOS}

De acuerdo a los resultados de la tabla 1 se evidencia que las variables están interrelacionadas dado que el nivel de significancia se ubica por debajo del nivel exigido $\left(\mathrm{p}=0,05^{* *}\right)$. La prueba de validación realizada, previa a la reducción de factores a través del análisis factorial, fue la medida de Kaiser - Meyer y Olkin (KMO) y la Prueba de esfericidad de Bartlett, determinando las medidas necesarias de comparación entre los coeficientes de correlación observados con los coeficientes de correlación parciales que puede asumir valores entre 0 y 1 . Teniendo en consideración un coeficiente de KMO $\geq 0,7$, los valores obtenidos de aproximación chi-cuadrado $(11876,540)$, gl (210) y significación $(0,000)$ el índice KMO determinado de 0,907 considerado muy bueno, ratifica que los datos pueden ser analizados mediante análisis factorial, que las dimensiones alcanzan grados de adecuación pertinentes y definen como pertinente el análisis factorial.

Sobre la base de los cinco factores relevantes determinados y que definen los rangos de decisión de los adultos mayores se puede afirmar que éstos adoptan sus decisiones considerando, diferencias cualitativas diferenciales entre estos factores, de acuerdo con la varianza explicada por cada uno de ellos y que totalizan un 72,656\%.

Los factores determinados fueron los siguientes: Factor 1: Características del producto, Factor 2: Salud y economía, Factor 3: Apariencia externa y marca, Factor 4: Procedencia y, finalmente Factor 5: Inocuidad del producto y calidad.

El Factor 1, denominado Características del producto, está conformado por los reactivos FA01 Color, FA02 Sabor, FA03 Tamaño, FA04 Olor, FA05 Frescura, FA06 Textura, componentes que ponen de manifiesto que los AM aprecian las características, principalmente de apariencia de los productos al momento de decidir su compra Se confirma de esta forma, con una varianza explicada de 42,348\%, la decisión que adoptan los AM, sin embargo, se aprecia que uno de los componentes, precisamente definido como FA07 Contenido nutricional, que alcanza una carga de 0,488, por ser ésta evaluada como baja, este componente fue excluido del análisis. Al parecer la información relevante que contiene el concepto de Contenido Nutricional, es un tipo de información sofisticada de difícil comprensión por parte de las personas, especialmente compleja a su vez para los adultos mayores, quienes, como se aprecia de los datos, no asignan suficiente valor, léase derivada de la carga factorial del componente, sobre algunos de los factores determinados en el estudio.

Complementando el análisis, el Factor 2, cualificado como de Salud y economía, estructurado sobre la base de los componentes FA08 Que sea beneficioso para la salud, FA19 Precio, FA20 Facilidad para obtenerlo y FA21 Rapidez para obtenerlo, permite apreciar cómo se estructura la decisión de compra de los AM, en esta caso, reconociendo que la decisión de compra combina el criterio de la salud a la par del económico como factores relevantes de la decisión. Se aprecia del análisis de los componentes de este segundo factor, que los adultos mayores aprecian en forma conjunta, por una parte el componente salud acompañado de la significancia que les reporta el componente economía. Se observa, en consecuencia, que si bien es cierto la economía del consumo de alimentos es relevante, lo es en también y, eventualmente, en la misma magnitud el componente salud, confirmando un factor de la mayor relevancia para los adultos mayores explicando el $10,7 \%$ de la varianza total. 
Tabla 1. Análisis de Factores de componentes principales en la elección de los alimentos por parte de los adultos mayores en la provincia del Guayas, Ecuador

\begin{tabular}{|c|c|c|c|c|c|}
\hline Componentes & $\begin{array}{l}\text { Características } \\
\text { del producto }\end{array}$ & $\begin{array}{l}\text { Salud y } \\
\text { economía }\end{array}$ & $\begin{array}{c}\text { Apariencia } \\
\text { externa y } \\
\text { marca }\end{array}$ & Procedencia & $\begin{array}{l}\text { Inocuidad del } \\
\text { producto y } \\
\text { calidad }\end{array}$ \\
\hline Color & 0,863 & & & & \\
\hline Sabor & 0,826 & & & & \\
\hline Tamaño & 0,736 & & & & \\
\hline Olor & 0,830 & & & & \\
\hline Frescura & 0,642 & & & & \\
\hline Textura & 0,711 & & & & \\
\hline Contenido nutricional & 0,488 & & & & \\
\hline Que sea beneficioso para la salud & & 0,539 & & & \\
\hline Precio & & 0,667 & & & \\
\hline Facilidad para obtenerlo & & 0,828 & & & \\
\hline Rapidez para obtenerlo & & 0,834 & & & \\
\hline Envase & & & 0,868 & & \\
\hline Etiqueta & & & 0,901 & & \\
\hline Marca & & & 0,831 & & \\
\hline Facilidad de preparación & & & & 0,578 & \\
\hline País de origen & & & & 0,845 & \\
\hline Región de Ecuador donde fue producido & & & & 0,831 & \\
\hline Respeto del medio ambiente durante la producción & & & & & 0,612 \\
\hline Forma de producción (orgánica o convencional) & & & & & 0,713 \\
\hline Sanidad & & & & & 0,691 \\
\hline Sello que garantice la calidad & & & & & 0,515 \\
\hline Varianza explicada \% & 42,348 & 10,767 & 8,098 & 6,217 & 5,226 \\
\hline
\end{tabular}

En la siguiente posición se ubica el Factor 3 bajo el título de Apariencia externa y marca, conformado por los componentes FA15 Envase, FA16 Etiqueta y FA17 Marca, permiten apreciar que los AM valoran, desde este punto de vista, la apariencia externa de los productos que adquiere y por supuesto, como se observa, valoran la seguridad y garantía que se expresa en el concepto "Marca", incluido en este factor. Respecto de este tercer factor, poca duda cabe que las personas, en general y por supuesto los adultos mayores en particular, aprecian en los productos que adquieren precisamente su apariencia, se expresada en su envase, tal vez atractivo o bien de uso fácil y práctico, como así también valoran la etiqueta que acompaña los productos que adquieren, sea también por su colorido, imagen que les reporta significación, como por el prestigio que la misma les transfiere. Todo esto se ve sintetizado, finalmente, en la marca del producto que, de una o de otra forma confirma en las personas la elección realizada al momento de adquirir sus productos alimenticios, es por ello que este factor termina por explicar un $8 \%$ de la varianza total dentro del estudio.

Se adiciona a este análisis el Factor 4, bajo la denominación Procedencia, incluyendo los reactivos FA09 Facilidad de preparación, FA10 País de origen y FA11 Región de Ecuador donde fue producido, mediante los cuales se ratifica el hecho que los AM también analizan y se percatan del origen y procedencia de los productos de alimentos que adquieren, en consecuencia, se constituye éste en un factor de importante relevancia para los productores, sus localizaciones y garantías de origen de sus productos. En este dimensión analizada. Se aprecia que los adultos mayores valoran por una parte, el país de origen de los productos que adquieren, eventualmente siguiendo los componentes de marca, calidad, imagen y prestigio que les reporta el consumo de productos de origen sofisticado, así como también asigna valor y reconocimiento a los productos ecuatorianos, en este caso apreciando la región de origen de los bienes que consumen, por cuanto el sentido regionalista de las personas se satisface en alto grado en este componente relevante dentro del factor aquí definido. 
Sin perjuicio de lo señalado, este factor integra a su vez un tercer componente, denominado facilidad de preparación, en referencia a la necesidad de los adultos mayores de lograr sus objetivos, con cierta facilidad, la cual les es otorgada por los bienes que consumen, precisamente bajo el concepto de "facilidad de preparación”. En definitiva este factor, es explicativo de un $6,2 \%$ de la varianza total por explicar.

Finalmente el último identificado fue el Factor 5, denominado Inocuidad del producto y calidad, se compone de los reactivos FA12 Respeto del medio ambiente durante la producción, FA13 Forma de producción (orgánica o convencional), FA14 Sanidad y FA18 Sello que garantice la calidad, pone de manifiesto que los AM valoran y aprecian las características, más bien intrínsecas que dan forma y contenido a los alimentos que adquiere, en consecuencia, las personas mayores, como se observa al analizar en detalle los componentes, asignan valor y reconocimiento a la inocuidad -Sanitización - y Sello de Calidad de los productos que adquieren llegando a explicar un 5,2\% de la varianza total por explicar en el presente estudio. Este último factor determinado, permite identificar ítems relevantes para los adultos mayores a la hora de decidir la compra de sus alimentos. Al respecto, el componente "Forma de producción (orgánica o convencional)" permite aquilatar la importancia que las personas asignan a este elementos de decisión, por una parte, por el valor que le asignan a la producción inocua de los productos como así también aprecian la vinculación de este elemento con otro ítem denominado "Respeto del medio ambiente durante la producción" conformando una dupla de componentes de alta relevancia para los adultos mayores, lo cual redunda en el sentido que tiene para las personas la "Sanidad" de los alimentos y que se les evidencia en el concepto de "Sello que garantice la calidad" de los productos alimenticios que decidan adquirir para su consumo.

\section{DISCUSIONES}

Sobre la base del análisis realizado, especialmente considerando las razones explicitadas que sostienen las decisiones de los adultos mayores en la adquisición de sus alimentos, se recomienda a las empresas extractivas, productoras industriales y comercializadoras que ofertan productos orientados al segmento de adultos mayores, que las áreas relacionadas con mercadeo y desarrollo de productos deben ser especialmente observados en sus decisiones.

El estudio realizado identifica y jerarquiza componentes clave de diseño y desarrollo de productos que, permiten a las empresas, asumir como orientadores y que se prevé que les permitan cubrir estas necesidades que resultan de interés para este grupo objetivo de mercado, denominado de adultos mayores. Desde la perspectiva del marketing de productos, resulta de gran importancia considerar que antes de anunciar un producto, se debe tomar en cuenta múltiples factores que pueden aportar significativamente a mejorar la compra de productos, de forma que las comunicaciones internas y externas de las empresas son clave, ayudarían a optimizarlos tiempos de búsqueda de criterios de diseño y producción además de colaborar en recoger información muy valiosa que definen las características generales de los productos, especialmente relevantes para este segmento de mercado de adultos mayores.

\section{CONCLUSIONES}

Luego del procesamiento y análisis de datos realizado en este trabajo, se concluye que: (a) Se determinó un sistema integrado de cinco factores que permite ratificar los criterios de decisión de los adultos mayores los cuales priorizan, en orden de rango de explicación, las Características del producto, la Salud y economía, la Apariencia externa y marca, la Procedencia y la Inocuidad del producto y calidad; (b) desde la perspectiva del marketing de productos, se concluye que existen características generales de los productos de alimentos apreciados por los adultos mayores, entre estos, las presentación externa, la marca y el sello de calidad que da la marca a estos productos; (c) desde la perspectiva de la estrategia de negocios, se hace evidente que existen componentes de diseño y de desarrollo de productos que el segmento de adultos mayores aprecia. Estas características fueron sintetizadas en los cinco factores determinados y constituyen una guía orientadora para definir estrategias de negocios eficaces; (d) se concluye, en torno a la relevancia de los hallazgos, para las unidades de marketing que recogen las 
necesidades y preferencias de sus clientes, definiendo las características generales de los productos y para los departamentos de producción que materializan en los productos las características técnicas específicas que definen los bienes y productos valorados por los clientes; y, para las áreas de gestión financiera, que analizan, asignan y regulan las fuentes de recursos, derivados de las ventas y los usos de los mismos aplicados en la producción.

\section{REFERENCIAS}

Aaker, David y George Dav (1996), Investigación de Mercados, McGraw-Hill, $3^{\circ}$ Ed., México.

Álvarez Munarríz Luis y Álvarez De Luis Amaia (2009). Estilos de Vida y Alimentación Gazeta de Antropología. 25(1). Disponible en: http://www.gazeta-antropologia.es/. $\mathrm{p}=1916$

Diener E, Suh E., Lucas R., Smiith H. (1999) Well-Being: three decades of progress. Psychol Bull. 125(2):276-302. Disponible en: http://doi.apa.org/getdoi.cfm?doi=10.1037/0033-2909.125.2.276

Eyssautier de la Mora Maurice (2006). Metodología de la Investigación. Quinta Edición. Editorial Thomson. México. 326 pp. Disponible en: http://cort.as/-SbX8 Leído en mayo 2019.

Forttes Valdivia Paula, Massad Torres Cristián y Cols (2009). Las Personas Mayores en Chile: Situaciones, Avances y Desafíos del envejecimiento. Servicio Nacional del Adulto Mayor. Naval. ISBN 978-956-8846-00-8. Disponible en: http://www.senama.gob.cl/storage/docs/Las-personas-mayores-de-chile-situacion-avances-desafios-del-envejecimiento-y-vejez-2009.pdf

Freiberg, H. A., Stover J. B., De la Iglesia G., (2013) Fernández M., Polychoric And Tetrachoric Correlations In Exploratory And Confirmatory Factorial Studies. Prensa Médica Latinoamericana - ISSN 1688-4094 Ciencias Psicológicas, VII (2): 151-164. https://www.redalyc.org/pdf/4595/459545415005.pdf

García Martín Miguel Ánglel (2002). El bienestar Subjetivo. Escritos de Psicología. 6: 18 - 39. Extraido de: https:/ dialnet.unirioja.es/servlet/articulo? ${ }^{\text {codigo }}=281674$

Garmendia, M. L. (2007). Análisis Factorial: una aplicación en el cuestionario de salud general de Goldbewrg. Revista Chile Salud Pública, 57-65.

Gonzalez, L. (1995). Sociedad de Consumo y Marketing. Madrid.

González, V. (2014). Estudio Hedónico del pan en el IES Mugardos. SGAPEIO, 1-25.

Grunert K, Dean D, Raats M, Nielsen N, Lumbers M. A measure of satisfaction with food-related life, Appetite 2007; 49(2): 486-93.

Hernández-Sampieri, R. \& Mendoza, C (2018). Metodología de la investigación. Las rutas cuantitativa, cualitativa y mixta, Ciudad de México, México: Editorial Mc Graw Hill Education, Año de edición: 2018, ISBN: 978-1-45626096-5, 714 p. http://virtual.cuautitlan.unam.mx/rudics/?p=2612

Johnson RA, Wichern DW. (2007). Applied Multivariate Statistical Analysis. Editorial Pearson Educación, Inc., USA. Disponible en: http://cort.as/-SbXK Leído en octubre 2019.

Kinnear C., Tomas; Taylor R., James, (1993), Investigación de Mercado un enfoque aplicado, Cuarta Edición. Mc Graw-Hill, 812 p.; pp 128-129, pp 311 -. 319, pp 414-415, pp 450-451, pp. 812.

Laudon, David y Albert Dellia Bitta (1991), Comportamiento del Consumidor, Prentice-Hall Hispanoamericana, $3^{\circ}$ Ed. 741p.p., México.

Leite Nacif , M., \& Ishimoto , E. (2001). Publicidad y Marketing la Información Nutricional. Brazil.

Likert R. A (1932) Technique for the Measurement of Attitudes, Archives of Psychology,140,1-55. Disponible en: http://cort.as/-SbXd Leído en mayo 2019

Mendoza, S. (2013). Factores determinantes de cumplimiento terapéutico en adultos mayores hipertensos. Santiago de Chile: Revista Médica de Chile.

Montoya, O. (2007). Aplicación del Análisis Factorial a la Investigación de Mercados, Caso de Estudio. Scientia et Technica Año XIII, 281-286.

Netuveli G, Blane D. (2008). Quality of life in older ages. Br Med Bull. 85(1):113-26.

Olivares, S., Lera M., L., \& Mardones, M. (2011). Promoción de alimentos y preferencias alimentarias en escolares. Chile.

Ortiz Arriagada Juana Beatriz (2009), Castro Salas Manuel. Bienestar psicológico de los adultos mayores, su relación con la autoestima y la autoeficacia: contribución de enfermería. Cienc. enferm. [Internet]. 15( 1 ): 25-31. Disponible en: http://dx.doi.org/10.4067/S0717-95532009000100004

Osorio P, Torrejón MJ, (2011) Anigstein MS. Calidad de vida en personas mayores en Chile. Revista Mad - Universidad de Chile. $\mathrm{N}^{\circ} 24,61-75 \mathrm{pp}$

Pérez, D., Rivera Márquez, J., \& Ortiz, L. (2010). Publicidad de alimentos en la programación de la televisión mexicana. Mexico.

Restrepo, S. (2006). Los Hábitos Alimentarios En El Adulto Mayor Y Su Relación Con Los Procesos Protectores Y Deteriorantes En Salud. Santiago de CHile: Revista Chilena de Nutrición.

Riveros, J., \& Berné, C. (2007). Análisis de la opinión de usuarios sobre calidad pericibida y satisfacción con hospital públicos: Estudio de caso desde la perspectiva de la aplicación del Marketing. Revista Médica Chile, $862-870$.

Rodrigues, A., \& Carmo, I. (2011). Asociación entre la comercialización de alimentos de densidad de alta energía y la obesidad infantil. Brazil.

Shamah-Levy, T. (2008). Estado de salud y nutrición de los adultos mayores en México: resultados de una encuesta probabilística nacional. México DF: Salud Pública de México.

Stanton, Williams Gruce Walker (1985), Fundamentos de Marketing, McGraw-Hill, 10ํE., México.

Urteaga, C. (2012). Validación del critrio de evaluación nutricional global del adulto mayor. Santiago de Chile: Revista Médica Chile

Walpole Ronald E., Myers Raymond H., Myers Sharon L., Ye Keying (2007). Probabilidad y estadística para ingeniería y ciencias. Novena edición. Editorial: Pearson.Disponible en: http://cort.as/-M8zq Leído en octubre 2019 . 